\title{
Functional Recovery of Aimed Scratching Movements after a Graded Proprioceptive Manipulation
}

\author{
Keri L. Page ${ }^{1}$ and Thomas Matheson ${ }^{1,2}$ \\ ${ }^{1}$ Department of Zoology, University of Cambridge, Cambridge CB2 3EJ, United Kingdom, and 2Department of Biology, University of Leicester, Leicester LE1 \\ 7RH, United Kingdom
}

To demonstrate the role of proprioceptive feedback in aimed limb movements, we induced graded changes in the signals provided by the principal receptor in a leg of a locust. The femoro-tibial chordotonal organ (FCO) of the hindleg monitors extension and flexion movements of the tibia and provides the main source of proprioceptive feedback about tibial kinematics. The FCO apodeme (tendon) was surgically shortened by different amounts to provide a systematic bias to this feedback, and aimed scratching movements were analyzed over the week after surgery. Shortening the apodeme led to increased firing of sensory neurons of the FCO at flexed joint angles and is thus functionally similar to flexing the tibia. Immediately after surgery, limb movements shifted dorsally and posteriorly, driven by overextension of the femoro-tibial joint and changes at other joints of the limb. The extent of tibial overextension reflected the extent of apodeme shortening. Overextension would tend to renormalize the FCO feedback signal and can be explained by known interjoint reflex pathways. Our data demonstrate that proprioceptive feedback provides a graded signal that is used to control these aimed limb movements. Over the course of $7 \mathrm{~d}$ after surgery, there was a functional recovery in aiming as the overall patterns of movement returned toward control values driven by reciprocal compensatory changes at two joints. The sensory to motor pathways are monosynaptic and oligosynaptic in this system, thus providing us with a powerful opportunity to investigate further the sensorimotor transformations and plasticity of aimed limb movements.

\section{Introduction}

In both vertebrates and invertebrates, proprioceptive sensory information can modify the performance of limb movements. Ghez and Sainburg (1995) showed that functionally deafferented patients could not account for joint interaction torques, so that elbow kinematics primarily reflected the mechanical effects of shoulder motion. Kargo and Giszter (2000) showed that ablation of muscle and joint afferents in the spinal frog led to changes in initial trajectory, effector path curvature, knee-joint velocity reversals, and overshooting misses of the target during aimed scratches. Hess and Büschges (1999) demonstrated that signals from a leg joint proprioceptor in a stick insect influenced motor neuronal activity of an adjacent limb joint. A key role of proprioceptive information is thus to regulate limb segment interactions. To date, it has not been possible to provide an experimentally graded bias to the proprioception of a limb joint to search for physiologically realistic effects on behavior. We make use of an insect model system in which we can surgically manipulate in a graded manner the key proprioceptive inputs from one joint of a limb. Our objectives were to, first, characterize the acute effects of

Received Jan. 8, 2009; revised Feb. 2, 2009; accepted Feb. 26, 2009.

This work was supported by a Biotechnology and Biological Sciences Research Council (BBSRC) studentship (K.L.P.) and a BBSRC advanced research fellowship and research grant (T.M.). We are grateful to Jure Zakotnik for writing the VideoTrack software, Volker Dürr for writing the Scratch Analysis software, and both Volker Dürr and Keir Pearson for providing valuable comments on a draft of this manuscript.

Correspondence should be addressed to Thomas Matheson, Department of Biology, University of Leicester, University Road, Leicester LE1 7RH, UK. E-mail: tm75@le.ac.uk.

DOI:10.1523/JNEUROSCI.0089-09.2009

Copyright $\odot 2009$ Society for Neuroscience $\quad$ 0270-6474/09/293897-11\$15.00/0 a graded bias of proprioception on aimed limb movements and, second, to determine whether the neuronal network controlling the limb showed a functional recovery of aiming.

In insects, movements and static positions of the tibia relative to the femur are encoded principally by feedback from one sense organ, the femoral chordotonal organ (FCO), which thus plays a key role in feedback control loops (Bässler, 1983; Field and Matheson, 1998). In quiescent animals, the reflex effects generally act as postural stabilizing mechanisms, but in active animals they can reverse in sign (Bässler, 1976, 1986). Ablating the FCO alters the performance of behaviors such as kicking (Jellema and Heitler, 1997), jumping (Bässler, 1967), and walking (Usherwood et al., 1968; Field, 2001). Surgically reversing the sign of proprioceptive feedback signaled by the FCO leads to abnormal (fully extended) leg postures in a stick insect (Bässler, 1979). In a fictively active stick insect preparation, FCO signals influence motor activity of several joints (Hess and Büschges, 1999). We demonstrate that an experimentally induced graded bias of proprioceptive signals from the FCO causes a graded error in aimed limb movements by affecting movements at several joints.

Both vertebrates and invertebrates show some functional recovery after perturbations of sensorimotor systems. Cats show adaptive changes in locomotor activity after de-efferentation (Bouyer et al., 2001), which is dependent on sensory feedback (Pearson et al., 2003), and locusts exhibit reorganization of function and underlying sensory neuropils after ablation of proprioceptive feedback in the flight system (Büschges et al., 1992). Sensory feedback from the FCO influences functional recovery after muscle damage in a stick insect leg (Bässler et al., 2007), but the 
disruptive effects of FCO feedback reversal per se do not diminish over time (Bässler, 1979; Bässler et al., 2007). In contrast, we show that the error in aiming caused by an experimental bias of FCO proprioceptive signals is corrected within $7 \mathrm{~d}$.

\section{Materials and Methods}

Video acquisition and analysis. Experiments were performed on 15 female locusts (Schistocerca gregaria; Forskål) taken from a crowded colony at the Department of Zoology, University of Cambridge (Cambridge, UK). Locusts were tethered in an experimental setup in the same way as described by Matheson and Dürr (2003). Tactile stimuli were applied to the posterior and anterior forewing randomly until at least 10 scratches were recorded for each of the stimulus sites. The locusts had 0.5- to 1-mmdiameter retroreflective discs (Scotchlite; $3 \mathrm{M}$ ) glued to their body and leg segments to permit video-based movement analysis (Zakotnik et al., 2004). The marked points included four positions on the hindleg to allow measurement of all joint angles. The animals were filmed from a lateral view, using a color digital video camera (TK-C1380; JVC) operated at a shutter speed of $1 / 500 \mathrm{~s}$. The images were taped on a super VHS video recorder (NV-HS9000; Panasonic), displayed on a monitor, and played back for capture using a personal computer video interface card (miroVIDEO DC30 plus; Pinnacle Systems).

Reflective markers were tracked using custom-written automated video-tracking software (Videotrack 2D version 1.0; programmed in Microsoft Visual C++) (Zakotnik et al., 2004). Movements were analyzed using Scratch Analysis software (programmed in Borland Delphi by Volker Dürr, University of Bielefeld, Bielefeld, Germany).

To describe and compare hindleg movements after different experimental procedures, the cyclical component of each grooming response (time, $>200 \mathrm{~ms}$ ) was quantified by a probability distribution $p(\mathrm{~T})$, describing the likelihood $p(\mathrm{X} \mid \mathrm{T})$ with which the distal tibia moved across point $\mathrm{X}$ in the workspace of the leg, given the treatment $\mathrm{T}$ (see Fig. 6) (Dürr and Matheson, 2003, their Methods). The probability distributions $p(\mathrm{~T})$ were determined separately for anteriorly and posteriorly directed scratches under different experimental conditions. The distributions were spatially smoothed using a Gaussian filter and normalized to a standard volume before being averaged across scratches. To assess differences between pairs of experimental conditions, the two pooled distributions were used as empirical likelihood functions. Bayes' theorem (Quinn and Keough, 2002) was then applied to determine which of the experimental conditions had most likely given rise to a movement in which the leg moved across a given point during a single video frame. By assuming a binomial distribution of $n$ independent observations and knowing the calculated error probability (formulae given by Dürr and Matheson, 2003), a critical number of observations was calculated that was necessary to allow no more than $n / 2-1$ wrong decisions in $95 \%$ of cases. If this critical value (in frames) was smaller than the average number of video frames in the total set of measured trajectories under consideration, the two distributions were considered statistically different. In other words, the movement distributions were considered to be significantly different if observation of a single scratch trajectory would be sufficient to distinguish them with $95 \%$ confidence.

FCO apodeme shortening. Preoperative (control) scratching was filmed as described above for 15 adult female locusts, after which each was secured laterally in Plasticene with the ipsilateral hindleg fully extended so that the lateral surface was uppermost. The FCO, located at the distal end of the femur, contains stretch- and relaxation-sensitive sensory neurons, the dendrites of which are embedded in a ligament (Fig. $1 \mathrm{~A}$, main ligament) that runs distally to insert onto a stiff cuticular tendon (FCO apodeme: Field, 1991) (Fig. 1A). This is an extension of the dorsal tibia. When the tibia is flexed, the apodeme pulls on the ligament, which becomes increasingly taut. When the tibia is extended, the apodeme is pushed proximally (allowing the main ligament to slacken) and then ventrally as the tibia rotates about its pivot point (Fig. $1 A$, asterisk) (Field, 1991; Shelton et al., 1992). When the tibia is fully extended (as in the secured locusts), the proximal attachment of the FCO apodeme to the tibia therefore lies relatively ventral in the leg, under the cover plate (Fig. 1B) (Bässler, 1979). A window was cut through the soft cuticle of the
A

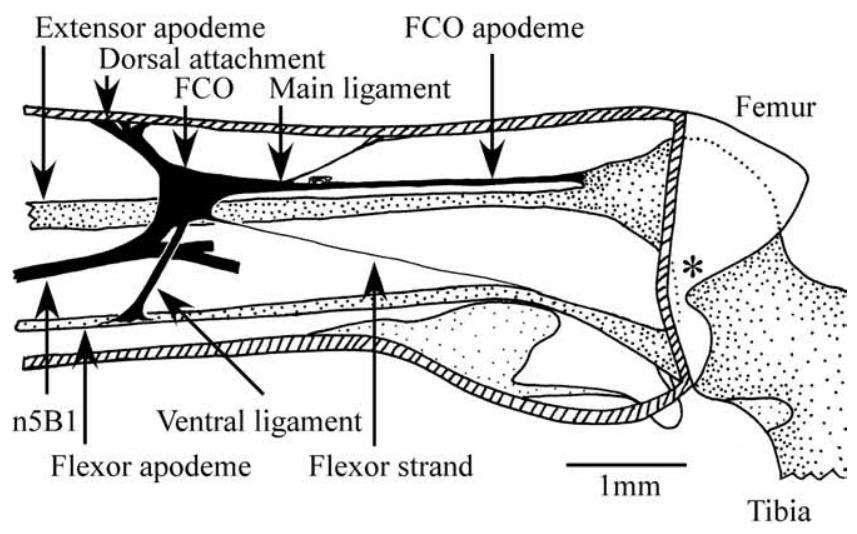

B

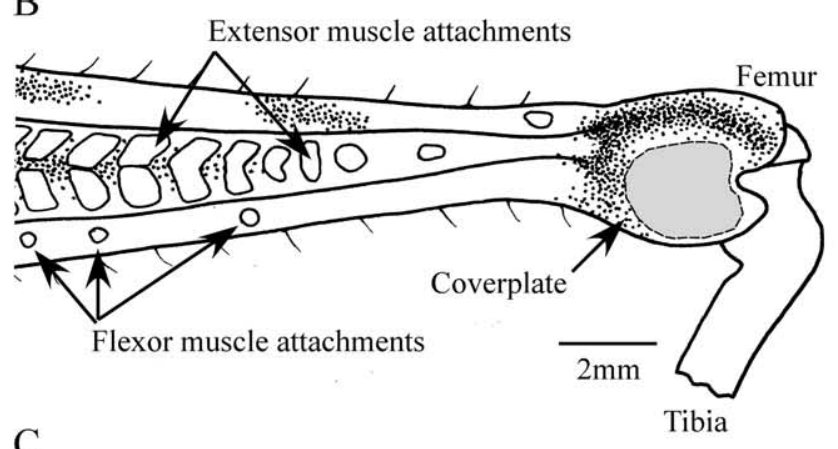

$\mathrm{C}$

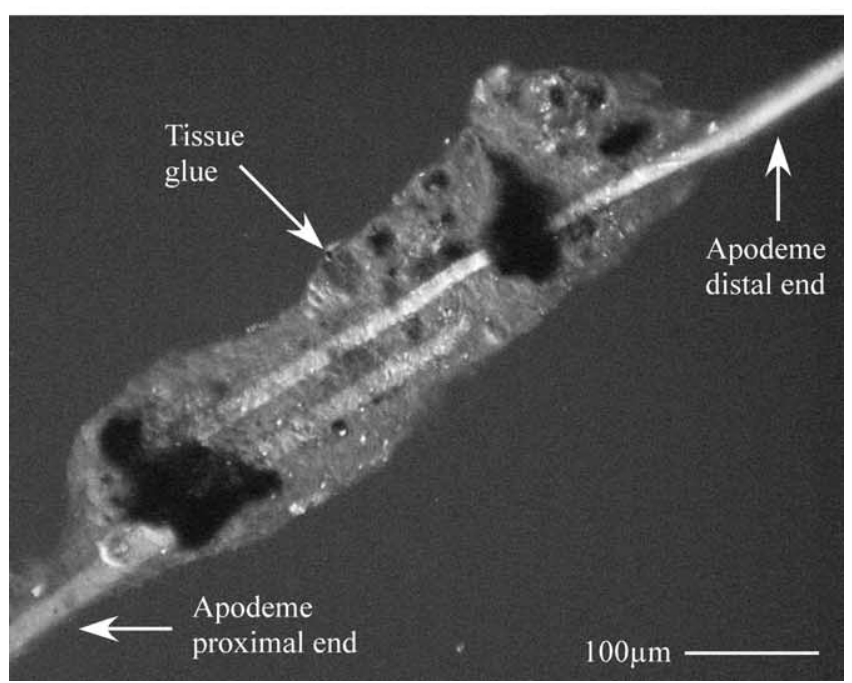

Figure 1. Illustrations of the distal metathoracic femur to show the location of the $\mathrm{FCO}(A, B)$ and a surgically shortened FCO apodeme ( $C$. $A$, Location of the FCO within the distal femur. Muscle fibers have been omitted for clarity. The FCO is anchored within the femur by a dorsal attachment strand and is attached to the tibia by three connections: the ventral ligament, the flexor strand, and the apodeme. The apodeme plays the key role in transmitting movements of the tibia to the dendrites of the FCO sensory neurons (Field, 1991; Shelton et al., 1992). The apodeme inserts onto a small protuberance of the tibia dorsal to the tibial extensor muscle apodeme, so that flexion movements of the tibia around the pivot point (asterisk) act to pull on the $\mathrm{FCO}$ apodeme and thus stretch the sensory dendrites. The $\mathrm{FC} 0$ is innervated by $\mathrm{n} 5 \mathrm{~B} 1$, which continues past the $\mathrm{FCO}$ to innervate a small number of exteroceptive sense organs farther distal in the leg (Matheson and Field, 1995). Nerve branches are omitted here for clarity. B, External view of the distal tibia illustrating the region of cuticle that was removed from the lateral cover plate to access the apodeme during surgery (gray shading). Points of attachment of muscle bundles are visible as light patches on the cuticle. C, Phase-contrast image of a surgically shortened apodeme taken at the end of the experimental period of $7 \mathrm{~d}$. Apodeme shortened by a length equivalent to $30^{\circ}$ of tibial flexion. 

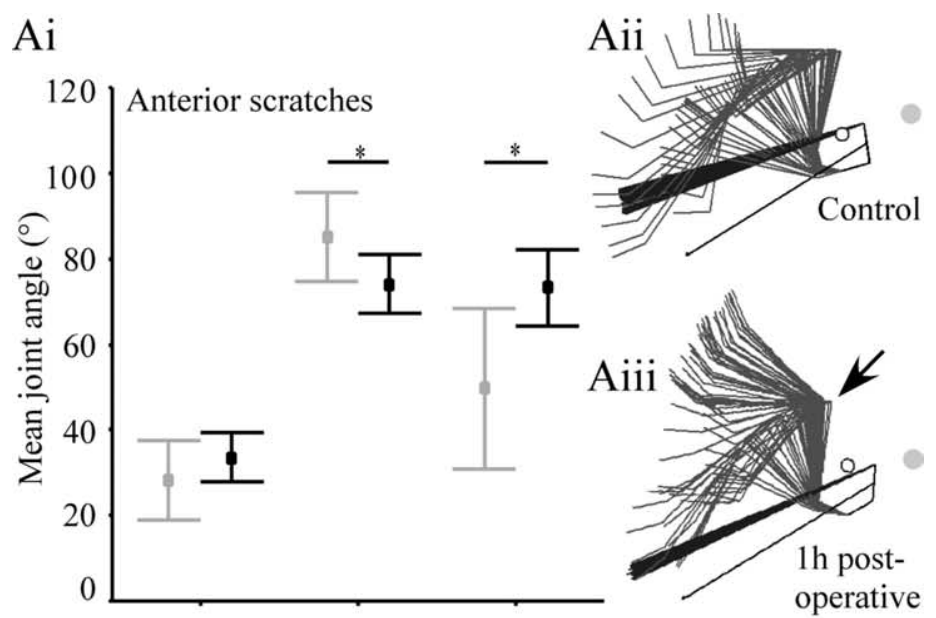

$\mathrm{Bi}$

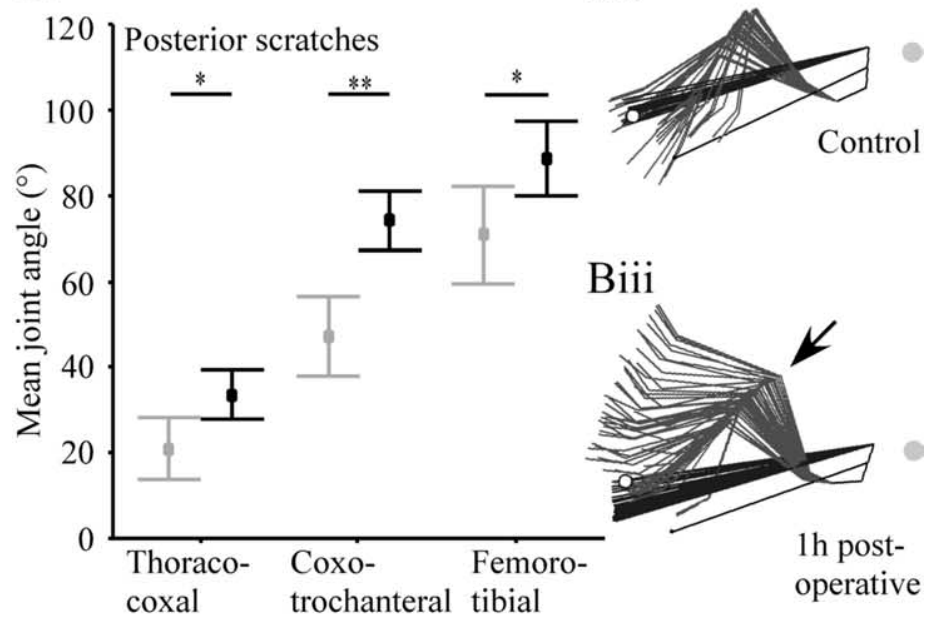

Joints

$\mathrm{C}$

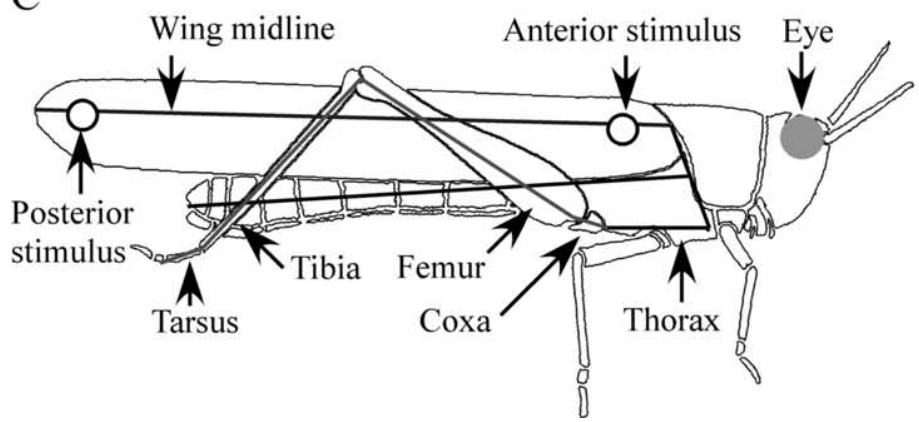

Figure 2. Joint angles for three of the hindleg joints before (gray) and $1 \mathrm{~h}$ after (black) shortening of the FCO apodeme. Apodeme shortening caused a significant increase in the mean femoro-tibial joint angle (i.e., tibial overextension) in both anterior $(\boldsymbol{A i})$ and posterior (Bi) scratches. In anterior scratches, there was also a significant decrease in the mean coxo-trochanteral joint angle but no change in thoraco-coxal joint angle $(\boldsymbol{A} \boldsymbol{i})$. In posterior scratches, there were significant increases in both the mean coxo-trochanteral and thoraco-coxal joint angles $(\boldsymbol{B i})$. Values are mean $\pm S D .{ }^{*} p<0.05 ;{ }^{* *} p<0.001$. The effect of surgery on the overall kinematics of scratching is indicated by the stick figures of scratching locusts (anterior scratches, Aii, Aiii; posterior scratches, Bii, Biii), in which leg and wing postures are represented at $20 \mathrm{~ms}$ intervals throughout a scratch (Cprovides a key to the stick diagrams). Postoperative shifts in joint angle (e.g., tibial overextension indicated by arrows in Aiiii and Biii) persisted throughout scratching movements. C, Schematic illustration of the correspondence between the stick figure representation of the locust and the named limb and body features.

cover plate (Fig. $1 B$, gray area) to reveal the apodeme that was then gripped in two places $(\sim 600 \mu \mathrm{m}$ apart) by fine forceps mounted on micromanipulators. In 10 locusts, the apodeme was then severed using microsurgical scissors, and the two cut ends were maneuvered so that the ends overlapped. This distance was measured using a calibrated eyepiece graticule. The overlapping ends were then glued together using Histoacryl tissue glue (B. Braun Aesculap) administered from the tip of a broken glass microelectrode (Fig. 1C). The shortened apodeme was then released from the forceps and observed through the cuticular window during tibial movement to ensure that the two ends remained bonded. All other FCO attachments were left intact, as were all trachea and air sacs. Performing the surgery as described avoided the need to cut through the heavily sclerotized ridges of cuticle of the dorsal distal tibia, which must remain intact to prevent the locust from breaking its own leg when jumping or kicking.

A sham operation was performed on five other female locusts in which a window was cut in the cuticle and glue was applied to the intact apodeme exactly as for the test animals. The only difference was that the apodeme was not shortened. In both cases, the cuticular window resealed naturally after hemolymph coagulation.

Shortening the FCO apodeme stretches the main ligament and is therefore functionally equivalent (in terms of activating FCO sensory neurons) to flexing the femoro-tibial joint. Calibrating the apodeme shortening in terms of femoro-tibial joint angle was performed by measuring the excursion of a marked point on an intact apodeme in micrometers during tibial rotation from 0 to $150^{\circ}$. This relationship is approximately linear for joint angles in this range (Field and Burrows, 1982). Apodemes were shortened by $160 \mu \mathrm{m}\left(17^{\circ}\right)$ in two locusts, 280 $\mu \mathrm{m}\left(30^{\circ}\right)$ in seven locusts, and $360 \mu \mathrm{m}\left(39^{\circ}\right)$ in a single animal. After surgery, the two locusts with a $17^{\circ}$ shortening, two with a $30^{\circ}$ shortening, and the single animal with a $39^{\circ}$ shortening were filmed at $1 \mathrm{~h}$. The latter animal autotomized its manipulated leg after filming at $1 \mathrm{~h}$. The remaining four were filmed again at the end of $1 \mathrm{~d}$, and these data were used to assess the relationship between the length of shortening and movement kinematics. The remaining five locusts, all of which had apodemes shortened by $30^{\circ}$, were filmed at $1 \mathrm{~h}, 1 \mathrm{~d}, 3 \mathrm{~d}, 6 \mathrm{~d}$, and $7 \mathrm{~d}$, to monitor acute effects and then any recovery or plasticity. The femoro-tibial joint was redissected at the end of the $7 \mathrm{~d}$ to check that the glued joint had remained intact. After initial surgery, each animal was lightly restrained by a loop of flexible wire passed around the pronotum and suspended at room temperature above a foam ball on which it could walk freely. Food was provided daily throughout the recovery period.

To rule out the possibility that the apodeme lengthened over the $7 \mathrm{~d}$ recovery period in response to the shortening procedure, an additional five sham and seven treated locusts were set up after first autotomizing the contralateral hindleg and measuring the length of its FCO apodeme. Apodeme length was measured from the point of attachment to the dorsal tibia to the proximal end of the stiff cuticular apodeme. In the treated animals, the remaining apodeme was shortened by $240 \mu \mathrm{m}\left(26^{\circ}\right)$, and, in shams, the remaining apodeme was exposed but not shortened. After $7 \mathrm{~d}$ of 
recovery, the remaining operated leg (for both sham and treated animals) was dissected open, and its FCO apodeme was measured. This length was subtracted from the corresponding control length obtained from the contralateral apodeme a week earlier, to estimate any change in length. These differences obtained from sham and treated groups were compared using a Student's $t$ test.

Extracellular recordings. Locusts were fixed ventral side up in Plasticene with one hindleg secured laterally in a fully extended position. The limb was secured so that both the lateral side was uppermost with the midfemur and femoro-tibial joint exposed for dissection. A window was cut laterally through the cuticle of the femoro-tibial joint, and the underlying tracheae were removed to expose the FCO apodeme. Branches of nerve $5 \mathrm{~B} 1$ (n5B1) distal to the FCO were severed using microsurgical scissors to remove sensory feedback from exteroceptors distal to the FCO (Burrows and Pflüger, 1988; Burrows, 1996). A second window was cut through the anterior surface of the midfemur to expose n5B1 proximal to the FCO. Nerve 5B2 was severed to prevent contraction of the tibial muscles and to remove sensory feedback from distal receptors (Burrows and Pflüger, 1988; Burrows, 1996). Nerve 5B1 was placed across a pair of extracellular hook electrodes and insulated using petroleum jelly to record the activity of FCO sensory neurons. Signals were recorded using standard extracellular amplifiers and captured to a computer using a CED1401 interface and Spike2 software (Cambridge Electronic Design). A total of 30 recordings were made from 10 animals. The tibia was moved from $90^{\circ}$ to $10^{\circ}$ to $170^{\circ}$ to $90^{\circ}$ in steps of $20^{\circ}$ and was held at each position for $30 \mathrm{~s}$ before recording the summed tonic firing rate of all detectable FCO neurons. After three trials through the full angular range in each animal, the FCO apodeme was shortened by $30^{\circ}$ using the method described above, and the responses of the FCO were recorded again as just described. The same protocol was conducted in three sham-operated animals.

Data were analyzed using SPSS version 10 . Values are reported as mean \pm SD unless stated otherwise.

\section{Results}

After shortening of the apodeme, locusts responded to touches on the wing with scratching movements that resembled qualitatively those made by intact animals and described in detail previously (Dürr and Matheson, 2003). We refer to movements made in response to touches at the anterior stimulus site (Fig. 2 Aii, C) as "anterior scratches" and those made in response to touches at the posterior stimulus site (Fig. 2Bii,C) as "posterior scratches," regardless of the kinematics of the movement.

\section{Shortening the FCO apodeme causes acute tibial overextension}

One hour after the chordotonal organ apodeme was shortened by a length equivalent to $30^{\circ}$ of tibial flexion, there was a significant increase in the mean femorotibial joint angle (i.e., tibial overextension) during both anterior and posterior scratches (Fig. $2 A i, B i$, Table 1). In anterior scratches, the mean femoro-tibial joint angle was increased from $50 \pm 19^{\circ}$ to $73 \pm 9^{\circ}$,
Table 1. Shortening the $\mathrm{FCO}$ apodeme by an amount equal to $30^{\circ}$ of tibial flexion had a significant effect on the mean femoro-tibial joint angles during both anterior and posterior scratches

\begin{tabular}{llcr}
\hline Stimulus & Joint & Anterior $F_{(4,98)}$, posterior $F_{(4,93)}$ & \multicolumn{1}{c}{$p$} \\
\hline Anterior & Thoraco-coxal & 1.21 & 0.30 \\
& Coxo-trochanteral & 5.19 & $<0.05$ \\
& Femoro-tibial & 6.51 & $<0.03$ \\
Posterior & Thoraco-coxal & 9.03 & $<0.02$ \\
& Coxo-trochanteral & 27.21 & 0.00 \\
& Femoro-tibial & 7.66 & $<0.02$ \\
\hline
\end{tabular}

Differences between control and postoperative scratches $(1 \mathrm{~h})$ are demonstrated by the significance level $(p)$ of univariate ANOVAs. The movements of all joints were significantly affected by the shortening, except for the thoraco-coxal joint during anterior scratches.
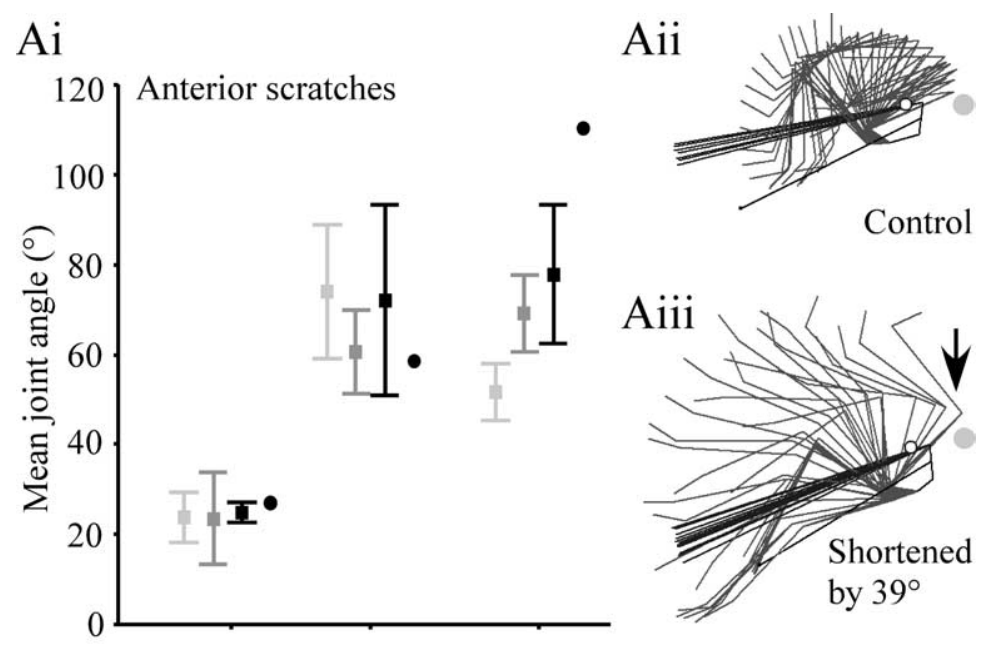

$\mathrm{Bi}$

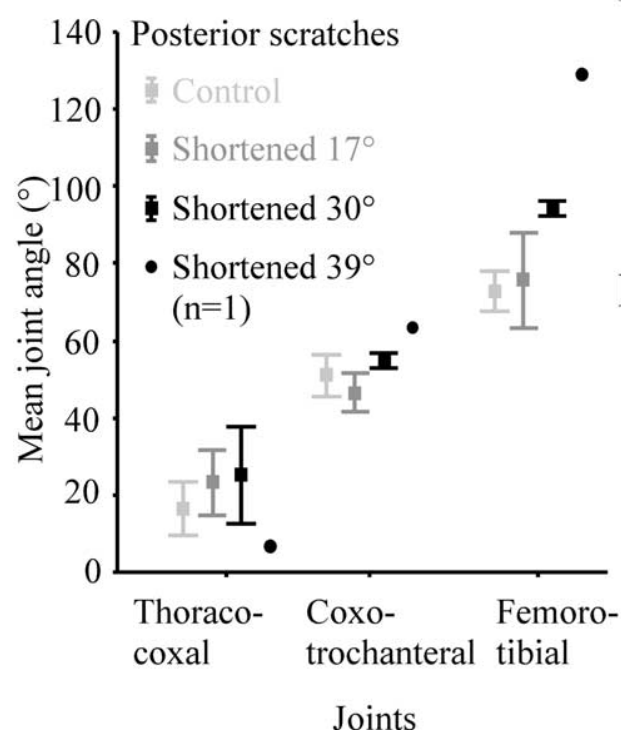

Bii

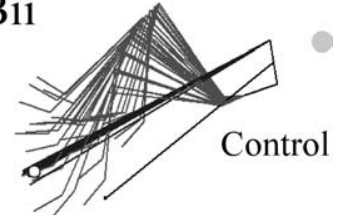

Biii

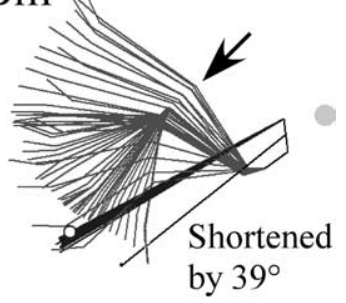

Figure 3. The relationship between the extent of apodeme shortening and mean joint angles for anterior scratches $(\boldsymbol{A})$ and posterior scratches $(\boldsymbol{B})$. $\boldsymbol{A}$, In anterior scratches the mean coxo-trochanteral angle decreased and the mean femoro-tibial joint angle increased with increased apodeme shortening. In posterior scratches, both the coxo-trochanteral and femoro-tibial joint angles increased with increased apodeme shortening. Small increases in the mean thoraco-coxal joint angle with increased apodeme shortening in both anterior and posterior scratches were not significant (see Results). The effect of shortening the apodeme by $39^{\circ}$ on the overall kinematics of scratching is indicated in Aii, Aiii, Bii, Biii). Postoperative shifts in joint angle (e.g., femoro-tibial overextension, arrows) persisted throughout the scratching movement. 


\section{A Anterior scratches. Mean scratch length 44 frames}

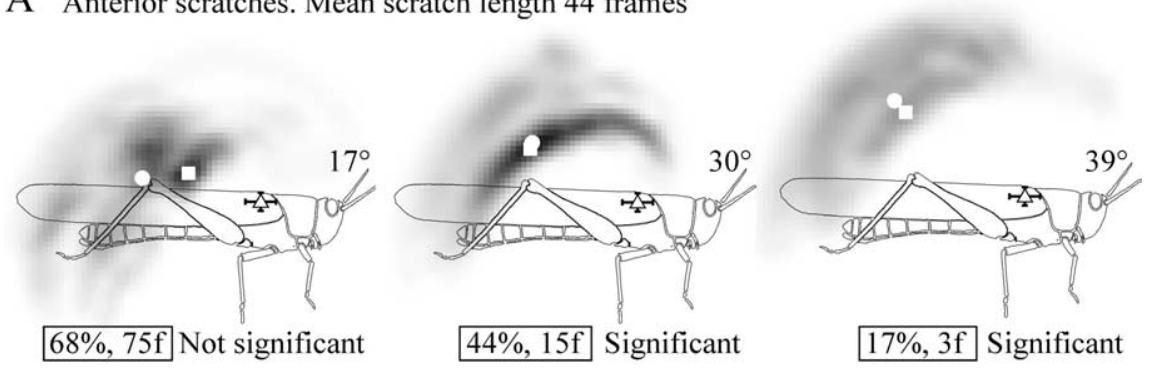

B Posterior scratches. Mean scratch length 39 frames

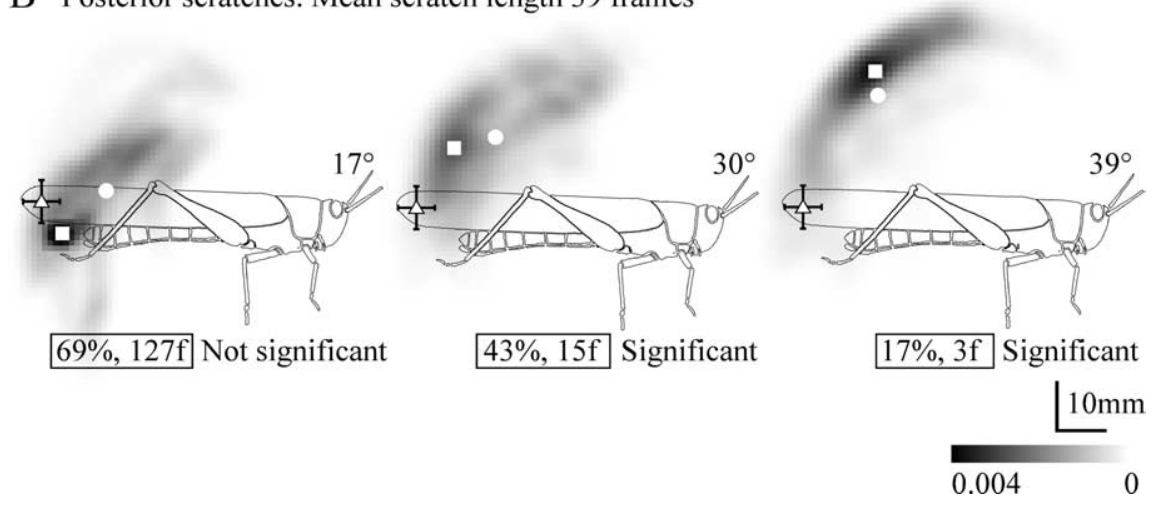

Figure 4. The effect of shortening apodeme length on movement distributions. The grayscale cloud represents the probability (per square millimeter; see scale bar) with which the distal tibia crossed any given point during anterior $(\boldsymbol{A})$ or posterior $(\boldsymbol{B})$ scratches. White circles are the centers of the distribution, and white squares are the most likely positions of the distal tibia (densest point). Numbers in boxes indicate the percentage overlap between distributions and the critical value, which is the number of video frames that would need to be analyzed to distinguish the distribution from control (larger numbers indicate more similar distributions; see Materials and Methods). Open triangles indicate the mean positions of the anterior and posterior stimuli ( $\pm 1 S D)$. Greater shortening led to progressively more dorsal distributions, with correspondingly smaller critical values.

$r_{(1,138)}=0.74, p<0.001$; anterior, $r_{(1,138)}$ $=0.70, p=0.001)$. The extent of shortening was negatively correlated with the mean coxo-trochanteral joint angle in anterior scratches (Spearman's $\rho$; $r_{(1,138)}=$ $-0.22, p<0.01$ ) and positively correlated with the mean coxo-trochanteral joint angle in posterior scratches (Spearman's $\rho$; $r_{(1,138)}=0.15, p<0.05$ ) (Fig. 3Ai,Bi).

There was no correlation between the extent of shortening and the mean thoraco-coxal joint angle in either anterior or posterior scratches (Fig. 3Ai,Bi) (Spearman's $\rho$; anterior, $r_{(1,138)}=0.01, p=0.44$; posterior, $\left.r_{(1,138)}=0.01, p=0.44\right)$. Shortening the apodeme by an amount equivalent to $39^{\circ}$ of flexion in one animal resulted in dramatic overextension of the tibia (Fig. 3 Aiii,Biii, arrows) during both anterior and posterior scratches (Fig. 3, compare Aii with Aiii, Bii with Biii).

To assess the combined effect of changes in all the leg joint angles on the overall scratching movement, we computed probability distributions for the location of the distal tibia throughout each movement. The similarity between pairs of these distributions is presented as a percentage overlap and as a critical value that is the number of video frames that would need to be observed to distinguish between them at a $95 \%$ confidence level (see Materials and Methods). When this critical value is smaller than the mean number of

whereas in posterior scratches, it increased from $71 \pm 11^{\circ}$ to $88 \pm$ $9^{\circ}$. In anterior scratches, there was also a significant decrease (from $85 \pm 10^{\circ}$ to $74 \pm 7^{\circ}$ ) in the mean coxo-trochanteral joint angle (Fig. 2Ai, Table 1). There was no effect on the thoraco-coxal joint angle. In posterior scratches, shortening the apodeme caused significant increases in both the mean thoraco-coxal joint angle (from $21 \pm 7^{\circ}$ to $34 \pm 6^{\circ}$ ) and the mean coxo-trochanteral joint angle (from $47 \pm 9^{\circ}$ to $74 \pm 7^{\circ}$ ) (Fig. 2 Bi, Table 1). The kinematics of postoperative scratches were qualitatively similar to those of control animals except that the hindleg joints moved through different angular ranges. In other words, the shifts in joint angle persisted throughout the whole movement rather than solely affecting (for example) just start or end postures (Fig. 2C,Aii,Aiii,Bii,Biii). Indeed, movements always began with the hindleg tarsus standing on a fixed platform, and locusts generally returned their tarsus to the ground or the platform between movements. There was no significant effect of the sham operation on any of the hindleg joints in either anterior or posterior scratches $1 \mathrm{~h}$ after surgery [multivariate ANOVA (MANOVA); anterior, $F_{(5,98)}=0.30, p=0.83$; posterior, $F_{(5,93)}=0.44, p=$ 0.73 ] or $1 \mathrm{~d}$ after surgery (MANOVA; anterior, $F_{(5,98)}=0.47, p=$ 0.71 ; posterior, $F_{(5,93)}=0.45, p=0.70$; data not shown).

To test whether apodeme shortening caused a graded alteration in aimed movements or a switch to a different movement strategy, apodemes were shortened by amounts corresponding to tibial flexions of 17,30 , and $39^{\circ}$. The extent of apodeme shortening was positively correlated with the mean femoro-tibial joint angle during scratches $1 \mathrm{~h}$ after surgery for both anterior (Fig. $3 A i)$ and posterior (Fig. 3Bi) scratches (Spearman's $\rho$; posterior, frames in the dataset of scratches being compared, we consider the distributions significantly different (Dürr and Matheson, 2003). Even for distributions that did not differ significantly on this criterion, both the percentage overlap and the critical values provide a measure of relative similarity, with larger values indicating more similar distributions.

Shortening the FCO apodeme by amounts equivalent to tibial flexions of 17,30 , or $39^{\circ}$ caused a progressive dorsal shift in the movement distribution of the distal tibia for scratches recorded $1 \mathrm{~h}$ after surgery (Fig. 4). This was reflected not only in the overall shapes of the distributions but also in the locations of the center of probability (Fig. 4, open circles) and the most likely position (Fig. 4, open squares). For both anterior (Fig. 4A) and posterior (Fig. $4 B$ ) scratches, the percentage overlap and critical values decreased as the extent of apodeme shortening increased, indicating progressively more different movements. The movement distributions for animals with apodemes shortened by 30 or $39^{\circ}$ were significantly different from those of controls (Fig. 4).

\section{Aimed limb movements recover within $7 \mathrm{~d}$}

To assess recovery in aimed limb movements after apodeme shortening, five animals with apodemes shortened by an amount equivalent to $30^{\circ}$ of tibial flexion were tested at time points of $1 \mathrm{~h}$, $1 \mathrm{~d}, 3 \mathrm{~d}, 6 \mathrm{~d}$, and $7 \mathrm{~d}$ after surgery.

Over the $7 \mathrm{~d}$ after apodeme shortening, there were significant changes in both the mean femoro-tibial joint angle and mean coxo-trochanteral joint angle with time (Fig. 5) (MANOVA; anterior, $F_{(5,29)}=3.60, p<0.005$; posterior, $F_{(5,29)}=13.43, p<$ $0.001)$. There was no significant change in the mean thoraco- 
A

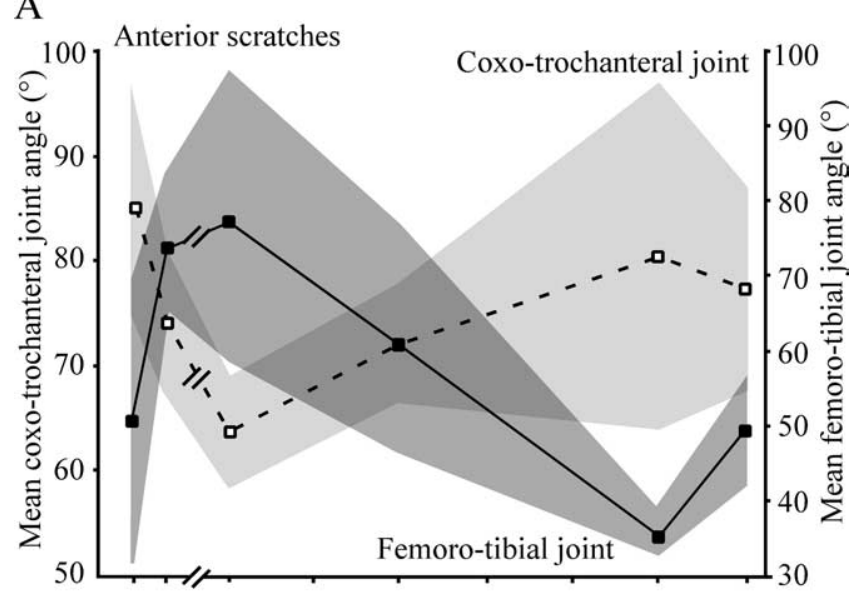

B

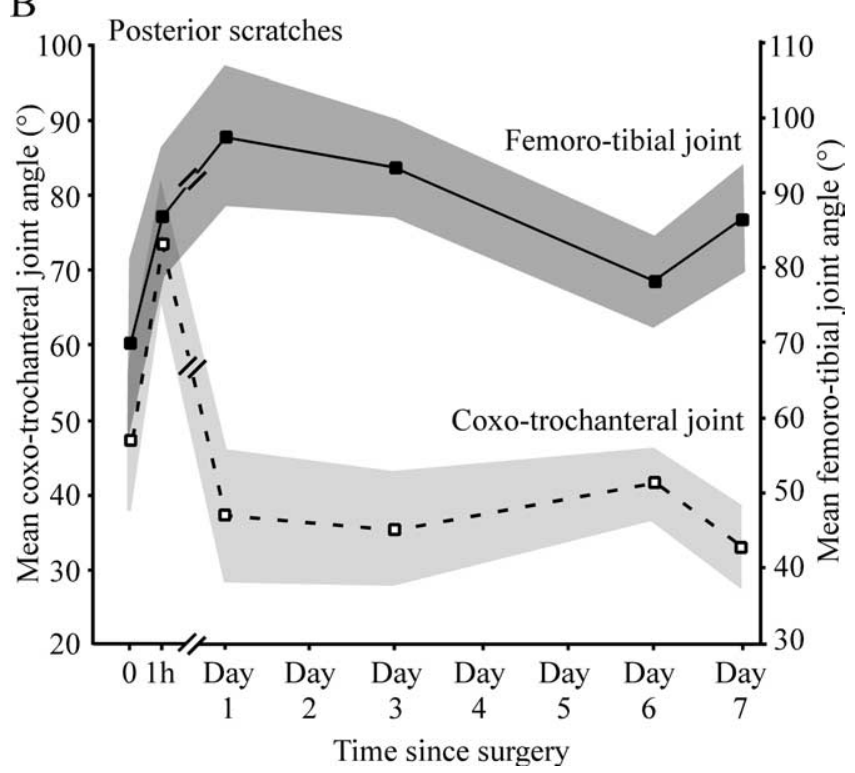

Figure 5. Mean coxo-trochanteral and femoro-tibial joint angles for scratches before $(0)$ and immediately after ( $1 \mathrm{~h}$ ) FCO apodeme-shortening surgery, and over the subsequent $7 \mathrm{~d}$. Mean joint angles had changed acutely for both joints within $1 \mathrm{~h}$ and then fluctuated over the following $7 \mathrm{~d}$ with a clear reciprocal relationship to one another. A key difference between anterior scratches $(\boldsymbol{A})$ and posterior scratches $(\boldsymbol{B})$ was the effect of surgery on the coxo-trochanteral joint angle at $1 \mathrm{~h}$ : in anterior scratches, the initial effect was a reduction in angle, whereas for posterior scratches, the effect was an increase. Dark and light gray shading indicate the SD.

coxal joint angle over the $7 \mathrm{~d}$ (ANOVA; anterior, $F_{(5,29)}=0.47$, $p=0.79$; posterior, $\left.F_{(5,29)}=2.17, p=0.09\right)$. A separate set of experiments demonstrated that there was no change in the length of the shortened apodeme over $7 \mathrm{~d}$ (Student's $t$ test, $t_{10}=-3.588$; $p=0.005$; data not shown).

In both anterior and posterior scratches, the mean femorotibial joint angle was greater $1 \mathrm{~h}$ after surgery than in controls (Fig. 5, filled symbols and lines) in accordance with our description above. There was an additional increase after $1 \mathrm{~d}$ and then a progressive fall back toward control levels at 3 and $6 \mathrm{~d}$ after surgery. For both anterior and posterior scratches, there was a small rebound on day 7 .

The pattern of change for coxo-trochanteral joint angle differed in the initial $1 \mathrm{~h}$ for anterior and posterior scratches but was similar for the remainder of the time (Fig. $5 A, B$, open symbols and dashed lines). For anterior scratches, the mean coxotrochanteral joint angle decreased during the first $1 \mathrm{~h}$, whereas for posterior scratches it increased. This initial rise in the coxotrochanteral joint angle was primarily responsible for the distinctive dorsal shift in the movement distribution evident in Figure $4 B$. In both anterior and posterior scratches, the mean coxotrochanteral angle decreased at day 1 to less than control values and then increased again by day 6 . There was a small rebound on day 7 (Fig. 5).

With the exception of the $1 \mathrm{~h}$ time point for posterior scratches, there were thus reciprocal decreases in coxotrochanteral joint angle corresponding to increases in femorotibial joint angle over time (Fig. 5). After $7 \mathrm{~d}$, the mean femorotibial joint angle for anterior scratches was within $2^{\circ}$ of control values and the coxo-trochanteral joint angle was within $8^{\circ}$ of controls (Fig. 5A). For posterior scratches the corresponding differences were 17 and $15^{\circ}$.

To assess the overall effect of these patterns of changes in joint angles over time, we computed movement probability distributions at each time point (Fig. 6). The overall control distributions, together with their centers of probability and most likely points are plotted in Figure 6, $A$ (anterior scratches) and $B$ (posterior scratches), together with the individual centers of probability for each of the five animals. The individual animal data all closely reflect the overall trend. One hour after apodeme shortening, the movement distribution for anterior scratches had shifted dorsally and posteriorly but was not significantly different from controls (critical value, 51 frames; average scratch duration, 48 frames). After $1 \mathrm{~d}$, the distribution was significantly different from controls, having shifted farther posteriorly and remaining dorsal relative to the controls (critical value, 43). From days 3 to 7 , the movement distribution shifted back toward (and was not significantly different from) the control distribution (Fig. 6A). This corresponded to an increase in the percentage overlap of the control and postoperative movement distributions from a minimum of $58 \%$ at $1 \mathrm{~d}$ to $76 \%$ on day 7 . On day 7 , the movement distribution could distinguished from controls in fewer than 307 frames.

The movement distribution of posterior scratches also shifted dorsally and posteriorly at $1 \mathrm{~h}$ (Fig. $6 \mathrm{~B}$ ) but was again not significantly different from controls (critical value, 31; average scratch duration, 30). From day 1 to 7 , the movement distribution shifted progressively back toward the control movement distribution (Fig. 6 B), and percentage overlap increased from 54 to $73 \%$. On day 7 , the two distributions could not be told apart in fewer than 123 frames.

In both anterior and posteriorly directed scratches, the process of compensation that occurred over the $7 \mathrm{~d}$ was the result of a posterodorsal shift in the movement distribution over the first $1 \mathrm{~h}$ (Fig. 6A, B, central illustrations), followed by a ventral and farther posterior shift by the end of day 1 . Between days 1 and 7 , an anteroventral shift occurred in both anterior and posterior scratches, bringing the centers of the movement distributions back toward the corresponding control distributions (Fig. 6A, $B$, central illustrations).

\section{Shortening the apodeme alters the response of the FCO}

To determine how the apodeme-shortening surgery affected the responses of sensory neurons in the FCO, we recorded overall tonic firing rates in control, sham, and operated animals. In control animals, the overall tonic firing rate of chordotonal afferents was lowest at a femoro-tibial angle of $70^{\circ}$ and increased toward both full flexion $\left(0^{\circ}\right)$ and full extension $\left(170^{\circ}\right)$ (Figs. $7 A, 8 A$ ). The responses we recorded came from many of the $\sim 90$ sensory neurons that comprise the FCO (Fig. 7) (Matheson and Field, 1990), 
A

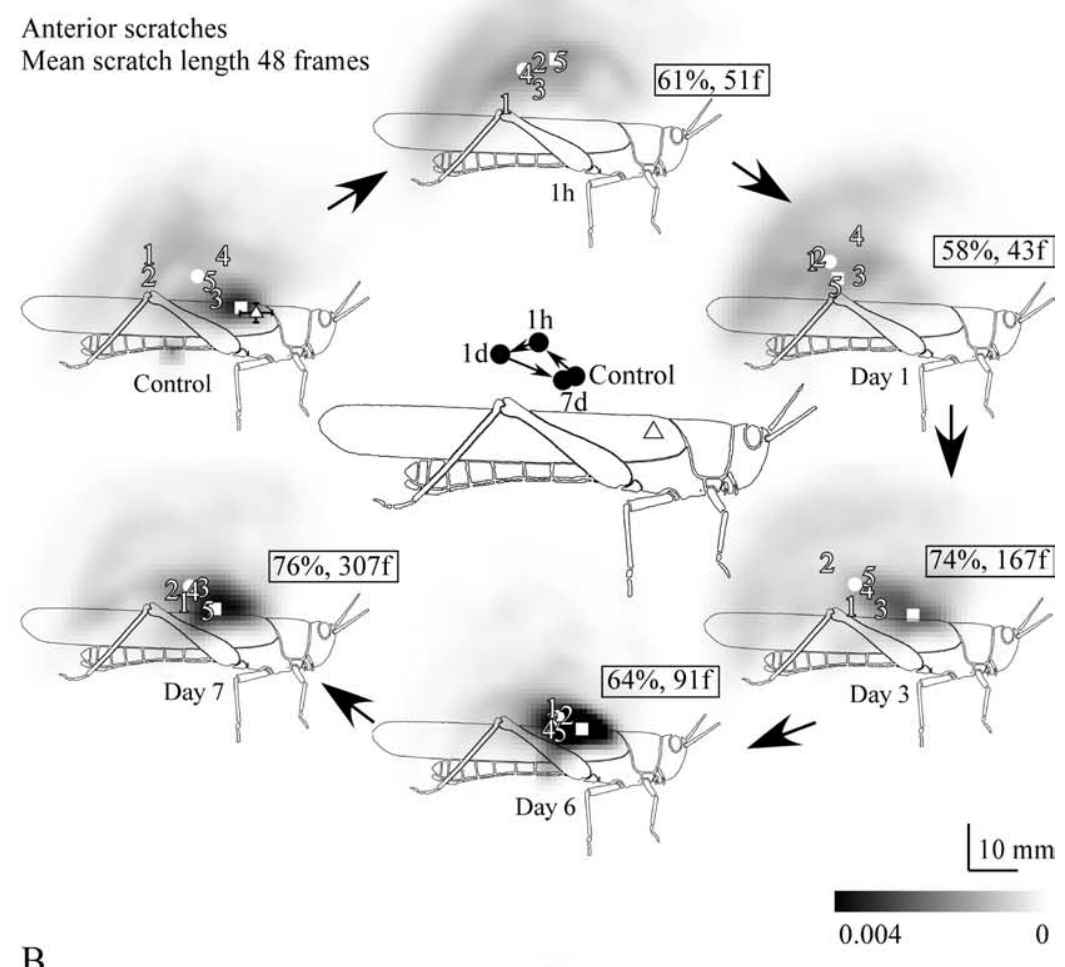

B

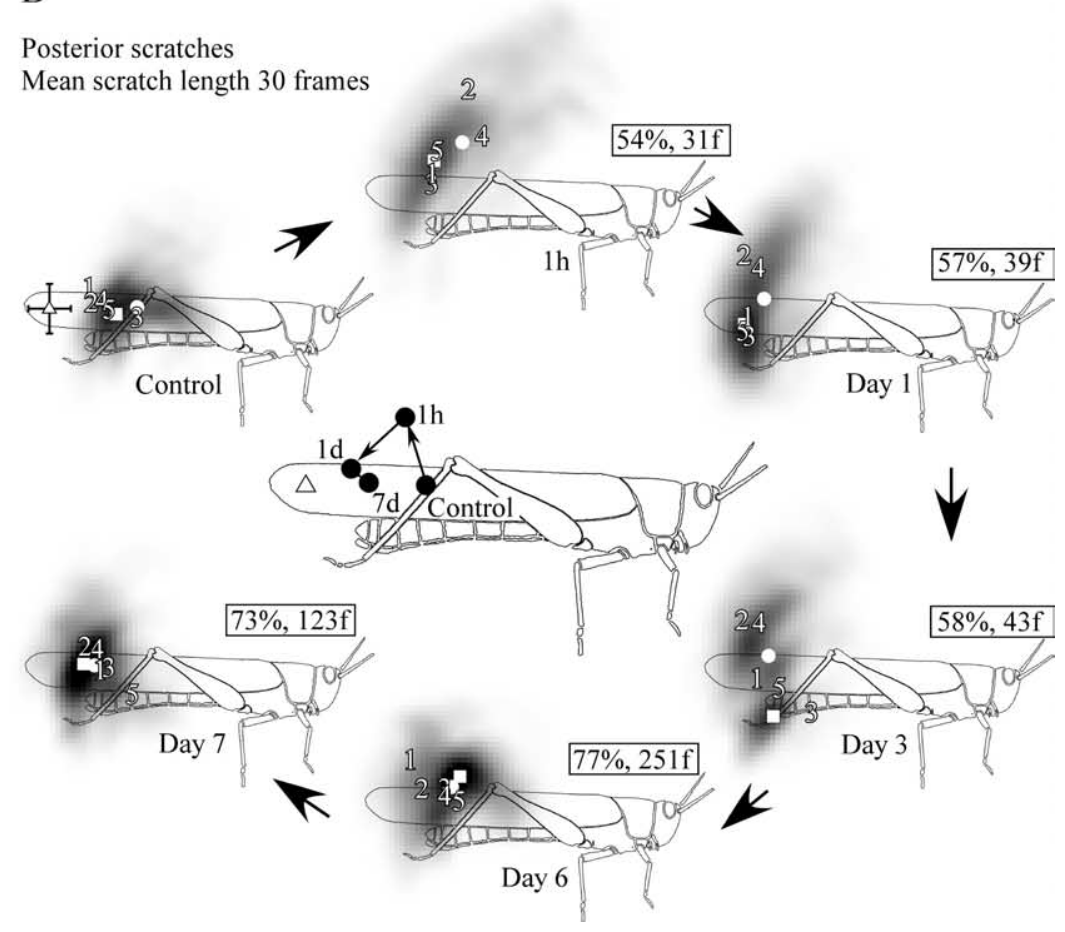

Figure 6. The shift in movement distributions relative to control for animals with apodeme shortening corresponding to $30^{\circ}$ of tibial flexion at $1 \mathrm{~h}, 1 \mathrm{~d}, 3 \mathrm{~d}, 6 \mathrm{~d}$, and $7 \mathrm{~d}$ after surgery (time course indicated by large arrows). $\boldsymbol{A}$, Anterior scratches. $\boldsymbol{B}$, Posterior scratches ( $n=5$ animals). Symbols as for Figure 4. Numbers $1-5$ indicate the individual centers of probability for each of the five animals represented in each overall movement distribution. Illustrations in the center of each time course summarize the acute shift in the overall centers of probability (filled circles) at $1 \mathrm{~h}$, followed by their progressive return to near the control locations. Small arrows indicate the direction of shift.

but we did not attempt to count or characterize the individual receptors. There was some hysteresis in the overall firing rate depending on whether the tonic activity was recorded after femoro-tibial extension or flexion (Fig. $8 A, B$, insets), so we av- eraged these responses to generate the comparisons shown in Figure $8 A-C$.

There was no difference between the mean overall activity of chordotonal afferents before and after the sham operation (Fig. $8 \mathrm{~A}$, all means within 1 standard deviation of corresponding controls). Shortening the apodeme by $30^{\circ}$ led to an increase in the mean overall firing rate at flexed angles of $\angle 90^{\circ}$ (Fig. 7 A, $B$, summary in Fig. $8 B$, Table 2, compare corresponding $10^{\circ}$ and $70^{\circ}$ traces). The activity recorded at extended femoro-tibial joint angles $\left(>90^{\circ}\right)$ was not significantly different from controls (Fig. $7 A, B$, summary in Fig. $8 B$, Table 2 , compare corresponding $170^{\circ}$ traces). When the small effect of sham operation was taken into account, the effect of apodeme shortening was a progressively greater enhancement of tonic firing for progressively more flexed tibial angles (Fig. 8C).

\section{Discussion}

We have shown that surgically shortening the apodeme of the femoro-tibial chordotonal organ causes a systematic alteration in the firing rate of its tonic sensory neurons. This is accompanied by graded changes in aimed limb movements that arise from alterations of joint kinematics at three joints of the limb. The changes in movement show functional recovery over $7 \mathrm{~d}$.

\section{The acute effects of FCO apodeme shortening}

After apodeme shortening, feedback from the manipulated FCO continued to be used by the joint control networks to regulate limb posture during scratching. Shortening the FCO apodeme by an amount equivalent to a flexion of $30^{\circ}$ caused a tibial overextension of $23^{\circ}$ during anterior scratches, an overextension of $17^{\circ}$ in posterior scratches (Fig. 2), and an increase in tonic receptor firing at flexed joint angles only, equivalent to that caused by an imposed tibial movement of $\sim 40^{\circ}$ (Fig. $8 C$ ). There was also a strong correlation between the length of the FCO apodeme and mean coxo-trochanteral joint angles (Figs. 2, 3). The observation that the mean behavioral effect was smaller than the manipulation is likely to be explained by the nonuniform effect of apodeme shortening on sensory firing across the full range of joint angles but could also indicate that biomechanical properties of the femur-tibia joint (Zakotnik et al., 2006; Page et al., 2008) stabilize its movements. Postoperative shifts in joint angles persisted throughout rhythmical scratching movements, indicating that FCO inputs were not 
gated out during the movement. Moreover, the effects were graded, so that increased shortening led to greater behavioral effects (Figs. 3, 4) rather than a simple shift to a different behavioral strategy or to an abnormal leg posture as seen when the FCO apodeme is surgically reconnected to the tibia so as to reverse completely the sign of its sensory feedback (stick insect: Bässler, 1979; Bässler et al., 2007).

The increase in mean femoro-tibial joint angle at $1 \mathrm{~h}$ can be explained by known reflex pathways. Shortening the apodeme mimics tibial flexion. Increased activity in afferents signaling a flexed position excites tibial extensor motor neurons and inhibits tibial flexor motor neurons through monosynaptic and oligosynaptic pathways (locust: Burrows, 1987, 1996; Field and Burrows, 1982). In the quiescent stick insect, such resistance (negative feedback) reflexes stabilize the position of the femoro-tibial joint against imposed movements (Bässler, 1974), whereas in an active animal, phase-dependent assistance (positive feedback) reflexes enhance ongoing movements (Bässler 1976; Cruse and Schmitz, 1983). One possibility is that resistance reflexes may be gated out to allow movements to proceed (locust: Burrows and Laurent, 1993), but some evidence suggests that they remain operational (stick insect: Cruse, 1981; Cruse and Pflüger, 1981). Our direct test of the normal role of proprioceptive feedback in a moving locust clearly shows that the "resistance" servomechanism (stick insect: Bässler, 1974) remains operational during scratching. That is, surgically shortening the apodeme led to a resistance-reflex-like overextension of the tibia not an assistance-reflex-like flexion.

Within the first $1 \mathrm{~h}$ after shortening the apodeme, there was increased levation of the coxo-trochanteral joint in posterior scratches but depression in anterior scratches (Fig. 5). We propose the following explanation for this contextdependent difference. Flexion of the femoro-tibial joint to angles below $90^{\circ}$ causes excitation of coxo-trochanteral levator motor neurons (stick insect: Hess and Büschges, 1999), so shortening the FCO apodeme should also provide a continuous sensory excitation of the coxotrochanteral levator motor neurons. This would explain the correlation between apodeme length and the observed increases in the mean angles of the coxotrochanteral and femoro-tibial joints during posterior scratches but cannot explain the coxo-trochanteral depression observed in anterior scratches. During the outward trajectory of normal anterior scratches, the coxo-trochanteral joint is levated much far-

\section{A Control}
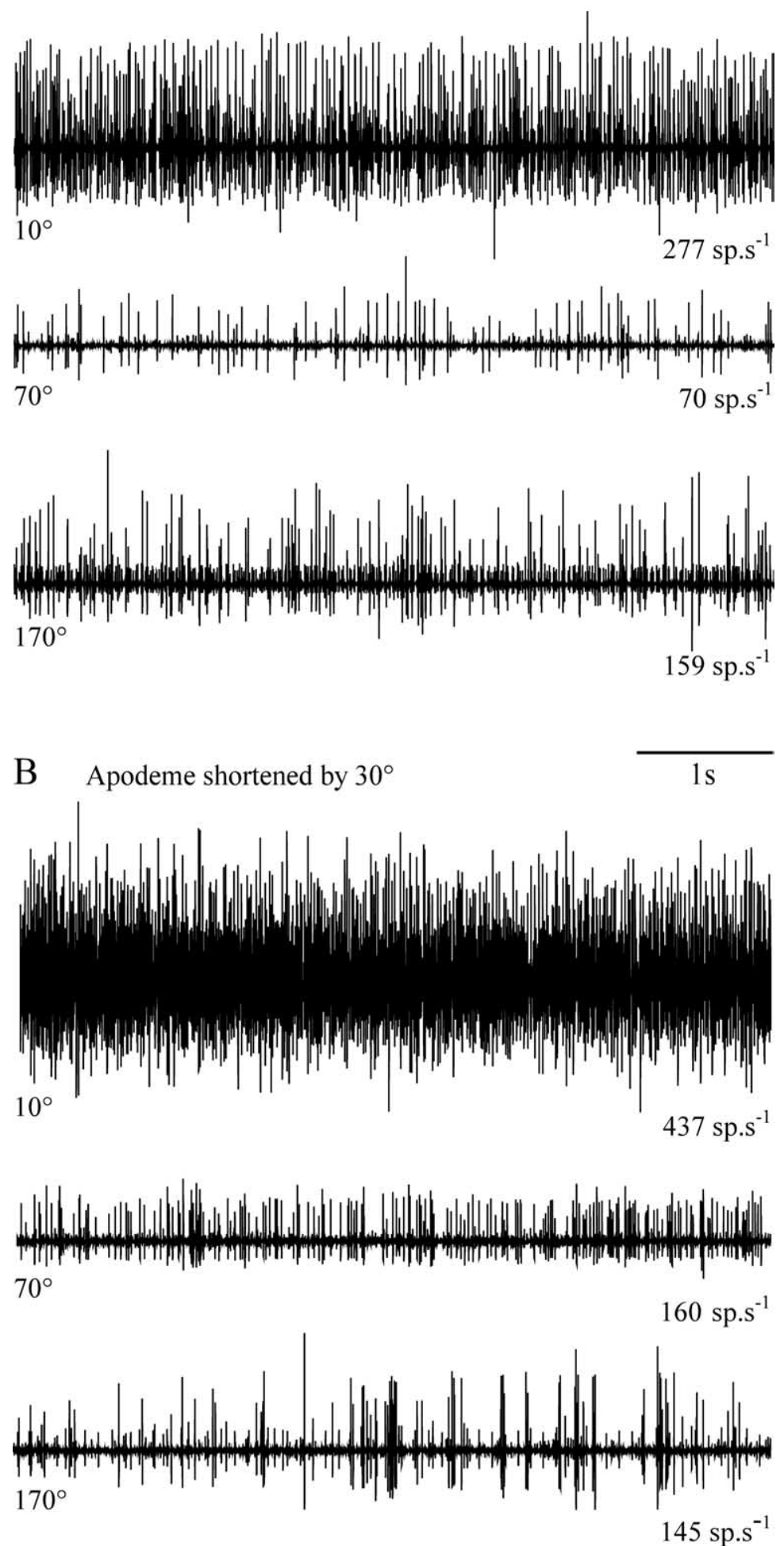

Figure 7. Extracellular recording of the activity of FCO sensory neurons at different angular positions of the femoro-tibial joint before $(\boldsymbol{A})$ and after $(\boldsymbol{B})$ apodeme shortening. All traces are from a single animal. The angular position of the tibia is indicated at the left of each trace and the mean spike frequency at the right. Shortening the apodeme caused an increase in activity at flexed joint angles (e.g., 10 and $70^{\circ}$ ) but not at extended joint angles (e.g., $170^{\circ}$ ).

ther than during posterior scratches (Fig. 5A, B, compare mean angles for controls). Proprioceptive sense organs on the trochanter, the hair row and hair plate, are activated by coxo-trochanteral levation, excite coxo-trochanteral depressor motor neurons and 
A
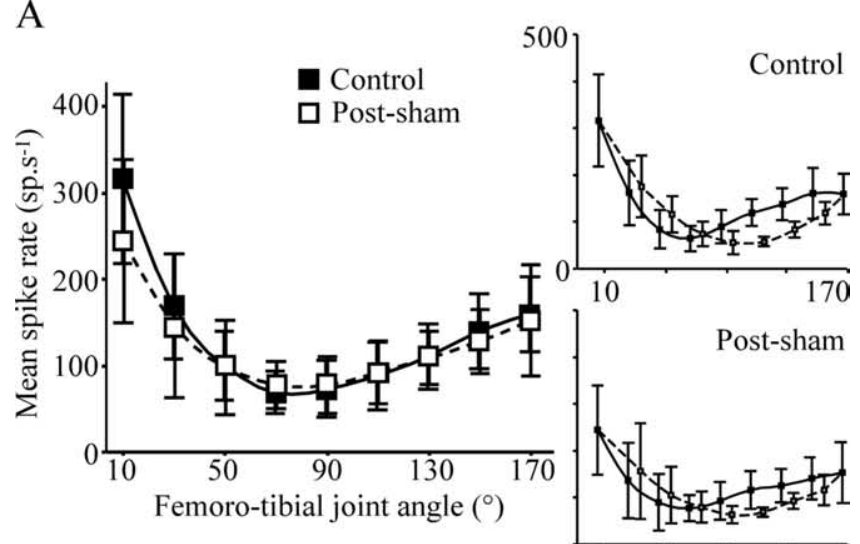

B

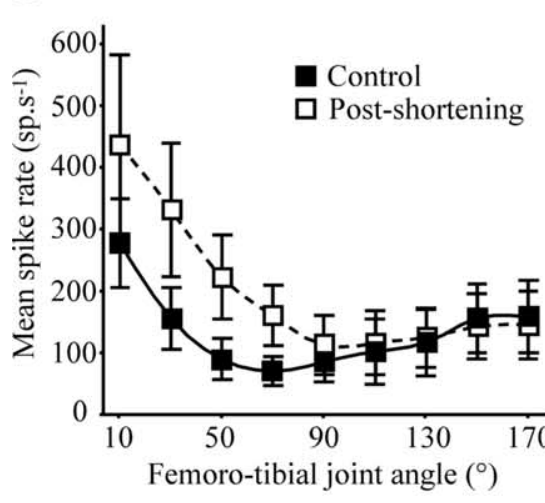

$\mathrm{C}$

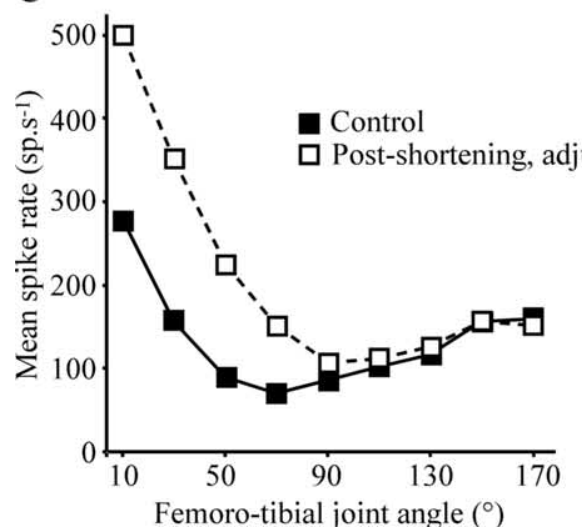

Figure 8. Mean spike rate of $\mathrm{FCO}$ sensory neurons before (solid lines and filled squares) and after (broken lines and open squares) a sham operation (A) or FCO apodeme shortening by $30^{\circ}$ (B). In control animals, the overall firing rate of $\mathrm{FCO}$ afferents was highest at flexed femorotibial angles $\left(10^{\circ}\right)$, decreased to a minimum at $70-90^{\circ}$, and increased again for more extended angles, in accordance with published data (Matheson, 1997). $\boldsymbol{A}$, Sham operation did not have a significant effect on the mean spike rate at any femoro-tibial joint angle. $\boldsymbol{B}$, Shortening the apodeme caused a significant increase in the mean spike frequency at femoro-tibial joint angles $<90^{\circ}$ but had no effect at joint angles above $90^{\circ}$. To provide the best estimate of the effect of apodeme shortening, the small effect of sham operation was removed from the "shortened" data (C). As reported previously (Zill, 1985), there was some hysteresis in the tonic response of FCO afferents depending on whether spike frequency was measured after extensions or flexions of the femoro-tibial joint (insets: filled symbols and solid lines, extensions; open symbols and broken lines, flexions). Responses measured after tibial extension and flexion were averaged to produce the curves in $\boldsymbol{A}-\boldsymbol{C}$.

inhibit coxo-trochanteral levator motor neurons (locust: Bräunig and Hustert, 1985a,b). It is possible that an interaction between sensory signals from the overextended tibia, and this negative feedback loop may contribute to depression of the coxotrochanteral joint in anterior scratches.
Table 2. Shortening the $\mathrm{FCO}$ apodeme had a significant effect on the mean tonic firing rate (spikes per second) recorded in nerve $5 \mathrm{~B} 1$ at relatively flexed femorotibial joint angles $\left(<90^{\circ}\right)$ but did not significantly affect the mean spike rate measured at relatively extended angles $\left(>90^{\circ}\right)$

\begin{tabular}{lcc}
\hline Femoro-tibial joint angle & $F_{(1,19)}$ (ANOVA) & $p$ \\
\hline 10 & 4.88 & $<0.05$ \\
30 & 21.44 & $<0.0001$ \\
50 & 30.68 & $<0.0001$ \\
70 & 27.89 & $<0.0001$ \\
90 & 2.17 & 0.16 \\
110 & 0.37 & 0.55 \\
130 & 0.11 & 0.74 \\
150 & 0.30 & 0.59 \\
170 & 0.15 & 0.71 \\
\hline
\end{tabular}

Functional recovery of aimed scratching movements

Durr and Matheson (2003) demonstrated that for the limb to reach different points along the midline of the wing requires a reciprocal relationship between femoro-tibial and coxotrochanteral joint angles (e.g., anterior targets require levated coxo-trochanteral angles and flexed femoro-tibial angles, whereas posterior targets require the opposite). Patterns of limb movement after apodeme shortening shifted dorsally and posteriorly, away from the target sites on the midline (Fig. 6). Functional recovery that brought the movement distributions back to near control locations (Fig. 6) occurred through reciprocal compensatory changes in both coxo-trochanteral and femoro-tibial joint angles (Fig. 5). The modifications of coxo-trochanteral joint angle presumably reflect interplay between experimentally biased interjoint reflex influences of the FCO (stick insect: Hess and Büschges, 1999) and intact intrajoint reflex effects of the many coxo-trochanteral joint proprioceptors (locust: Bräunig and Hustert, 1985a,b; Burrows, 1996). Mean femoro-tibial joint angle increased, decreased, and then increased again over the $7 \mathrm{~d}$ of recovery, whereas coxo-trochanteral joint angles followed the inverse pattern. There is a similar oscillation in the recovery of escape behavior of crickets after cercal ablation (Kanou et al., 1999). Normal movements of the femoro-tibial joint are controlled through an interplay between motor activity and biomechanical properties of the limb (Zakotnik et al., 2006; Page et al., 2008). For example, passive forces tend to return the tibia to a position near the middle of its working range in the absence of motor drive. The complex patterns of recovery seen at several joints of the limb after FCO surgery suggest that a simple biomechanical explanation for the recovery is unlikely. It is not known whether the biomechanical properties are themselves subject to plasticity.

Within $7 \mathrm{~d}$, movement distributions and mean joint angles were both close to control values for anterior scratches (Figs. 5, 6). Over the same period, movement distributions for posterior scratches recovered somewhat less, remaining posterior to control distributions, and joint angles had also not yet returned completely to control values. Recovery of cricket escape behavior from cercal ablation takes 6-14 d (Kanou et al., 1999), and recovery of locust flight from tegula ablation takes 14-21 d (Gee and Robertson, 1996; Wolf and Büschges, 1997). In striking contrast, however, locusts and stick insects do not recover from complete reversal of the sign of proprioceptive feedback from the FCO within 2 months (Bässler, 1979). The femoro-tibial joint remains abnormally extended, and the basal joints are elevated so the leg is held in a "salute" posture, with transitions between saluting and more normal limb movements occurring in the walking stick 
insect (Graham and Bässler, 1981). In resting stick insects, an abnormal leg waving behavior that is seen in the first few days after FCO reversal diminishes over time but can still occur after $30 \mathrm{~d}$ (Bässler et al., 2007). Together, these data suggest that functional recovery is possible for marked biases of FCO proprioceptive input, but that the underlying neuronal plasticity cannot compensate for a complete reversal of the sign.

\section{Recalibration of the FCO feedback control loop}

Functional recovery of aiming after apodeme shortening must require either recalibration of FCO inputs against an error signal or homeostatic regulation of the firing of FCO neurons or their central synaptic outputs. Possible error signals intrinsic to the limb include a mismatch between those of the FCO and the femoro-tibial strand receptor that responds to tibial extension (locust: Bräunig, 1985), the accessory flexor tension receptor that responds to accessory flexor muscle force (locust: Matheson and Field, 1995), or multipolar joint receptors that signal tibial movements (locust: Coillot and Boistel, 1969; Heitler and Burrows, 1977; Williamson and Burns, 1978; Matheson and Field, 1995; stick insect: Bässler, 1977). FCO sensory neurons may be subject to indirect presynaptic inhibition by these other proprioceptors during tibial movements, thus modulating the gain of FCO feedback during recovery. FCO sensory neurons in the stick insect are modulated presynaptically by pathways from exteroceptors (Stein and Schmitz, 1999), but it is not known whether similar pathways exist from proprioceptors.

Locusts could also use error signals extrinsic to the leg in recalibration. They could detect the location of the end point of the tibia during a scratch if it touched sensory hairs on the wing surface (Page and Matheson, 2004), or they could detect erroneous contact with the substratum during locomotion through ground reaction forces and interactions with the other legs.

Acutely altered firing of FCO sensory neurons, or the strengths of their central synaptic outputs, could return to control levels through mechanical-, cellular-, or network-level homeostatic mechanisms. We have demonstrated that the cuticular apodeme is not a site of mechanical adaptation, but the possibility remains that the ultrastructure of the receptor cells or their attachments within the FCO are subject to mechanical changes during recovery. In the stick insect, experimental reversal of FCO signals leads to a long-term decrease in the gain of reflexes mediated by the organ (Bässler et al., 2007), suggesting that homeostatic mechanisms might be at play. We have demonstrated that, in the locust, a similar but graded change in FCO signaling is compensated for within $7 \mathrm{~d}$ so that initially misdirected scratches regain their accuracy.

\section{References}

Bässler U (1967) Zur Regelung der Stellung des Femur-Tibia-Gelenkes bei der Stabheuschrecke in der Ruhe und im Lauf. Kybernetik 4:18-26.

Bässler U (1974) Vom femoralen Chordotonalorgan gesteuerte Reaktionen bei der Stabheuschrecke Carausius morosus: Messung der von der Tibia erzeugten Kraft im aktiven und inaktiven Tier. Kybernetik 16:213-226.

Bässler U (1976) Reversal of a reflex to a single motoneurone in the stick insect Carausius morosus. Biol Cybern 24:47-49.

Bässler U (1977) Sense organs in the femur of the stick insect and their relevance to the control of position of the femur-tibia joint. J Comp Physiol 121:99-113.

Bässler U (1979) Effects of crossing the receptor apodeme of the femoral chordotonal organ on walking, jumping and singing in locusts and grasshoppers. J Comp Physiol 134:173-176.

Bässler U (1983) Neural basis of elementary behaviour in stick insects. Berlin: Springer.

Bässler U (1986) Afferent control of walking movements in the stick insect
Carausius morosus. II. Reflex reversal and the release of the swing phase in the restrained foreleg. J Comp Physiol A Neuroethol Sens Neural Behav Physiol 158:351-362.

Bässler U, Wolf H, Stein W (2007) Functional recovery following manipulation of muscles and sense organs in the stick insect leg. J Comp Physiol A Neuroethol Sens Neural Behav Physiol 193:1151-1168.

Bouyer LJ, Whelan PJ, Pearson KG, Rossignol S (2001) Adaptive locomotor plasticity in chronic spinal cats after ankle extensors neurectomy. J Neurosci 21:3531-3541.

Bräunig P (1985) Strand receptors associated with the femoral chordotonal organs of locust legs. J Exp Biol 116:331-341.

Bräunig P, Hustert R (1985a) Actions and interactions of proprioceptors of the locust hind leg coxo-trochanteral joint. I. Afferent responses in relation to joint position and movement. J Comp Physiol A Neuroethol Sens Neural Behav Physiol 157:73-82.

Bräunig P, Hustert R (1985b) Actions and interactions of proprioceptors of the locust hindleg coxo-trochanteral joint. II. Influence on the motor system. J Comp Physiol A Neuroethol Sens Neural Behav Physiol 157:83-89.

Burrows M (1987) Parallel processing of proprioceptive signals by spiking local interneurons and motor neurons in the locust. J Neurosci 7:1064-1080.

Burrows M (1996) The neurobiology of an insect brain, p 682. Oxford: Oxford UP.

Burrows M, Laurent G (1993) Synaptic potentials in the central terminals of locust proprioceptive afferents generated by other afferents from the same sense organ. J Neurosci 13:808-819.

Burrows M, Pflüger HJ (1988) Positive feedback loops from proprioceptors involved in leg movements of the locust. J Comp Physiol 163:425-440.

Büschges A, Ramirez JM, Driesang R, Pearson KG (1992) Connections of the forewing tegulae in the locust flight system and their modification following partial deafferentation. J Neurobiol 23:44-60.

Coillot JP, Boistel J (1969) Étude de l'activité électrique propagée des récepteurs à l'étirement de la patte métathoracique du criquet, Schistocerca gregaria. J Insect Physiol 15:1449-1470.

Cruse H (1981) Is the position of the femur-tibia joint under feedback control in the walking stick insect? I. Force measurements. J Exp Biol 92:87-95.

Cruse H, Pflüger HJ (1981) Is the position of the femur-tibia joint under feedback control in the stick insect? II. Electrophysiological recordings. J Exp Biol 92:97-107.

Cruse H, Schmitz J (1983) The control system of the femur-tibia joint in the standing leg of a walking stick insect Carausius morosus. J Exp Biol 102:175-185.

Durr V, Matheson T (2003) Graded limb targeting in an insect is caused by the shift of a single movement pattern. J Neurophysiol 90:1754-1765.

Field LH (1991) Mechanism for range fractionation in chordotonal organs of Locusta migratoria (L) and Valanga sp. (Orthoptera: Acrididae). Int J Insect Morphol Embryol 20:25-39.

Field LH (2001) Neuromuscular physiology and motor control. In: The biology of wetas, king crickets and their allies (Field LH, ed), pp 459-489. Wallingford, CT: CAB International.

Field LH, Burrows M (1982) Reflex effects of the chordotonal organ upon leg motor neurons of the locust. J Exp Biol 101:265-285.

Field LH, Matheson T (1998) Chordotonal organs of insects. Adv Insect Physiol 27:1-228.

Gee C, Robertson R (1996) Recovery of the flight system following ablation of the tegulae in immature adult locusts. J Exp Biol 199:1395-1403.

Ghez C, Sainburg R (1995) Proprioceptive control of interjoint coordination. Can J Physiol Pharmacol 73:273-284.

Graham D, Bässler U (1981) Effects of afference sign reversal on motor activity in walking stick insects (Carausius morosus). J Exp Biol 91:179-193.

Heitler WJ, Burrows M (1977) The locust jump. II. Neural circuits of the motor programme. J Exp Biol 66:221-241.

Hess D, Büschges A (1999) Role of proprioceptive signals from an insect femur-tibia joint in patterning motoneuronal activity of an adjacent leg joint. J Neurophysiol 81:1856-1865.

Jellema T, Heitler WJ (1997) The influence of proprioceptors signalling tibial position and movement on the kick motor programme in the locust. J Exp Biol 200:2405-2414.

Kanou M, Ohshima M, Inoue J (1999) The air-puff evoked escape behav- 
iour of the cricket Gryllus bimaculatus and its compensational recovery after cercal ablations. Zool Sci 16:71-79.

Kargo WJ, Giszter SF (2000) Afferent roles in hindlimb wipe-reflex trajectories: free-limb kinematics and motor patterns. J Neurophysiol 83:1480-1501.

Matheson T (1997) Octopamine modulates the responses and presynaptic inhibition of proprioceptive sensory neurones in the locust Schistocerca gregaria. J Exp Biol 200:1317-1325.

Matheson T, Dürr V (2003) Load compensation in targeted limb movements of an insect. J Exp Biol 206:3175-3186.

Matheson T, Field LH (1990) Innervation of the metathoracic femoral chordotonal organ of Locusta migratoria. Cell Tissue Res 259:551-560.

Matheson T, Field LH (1995) An elaborate tension receptor system highlights sensory complexity in the hindleg of the locust. J Exp Biol 198:1673-1689.

Page KL, Matheson T (2004) Wing hair sensilla underlying aimed hindleg scratching of the locust. J Exp Biol 207:2691-2703.

Page KL, Zakotnik J, Dürr V, Matheson T (2008) Motor control of aimed limb movements in an insect. J Neurophysiol 99:484-499.

Pearson KG, Misiaszek JE, Hulliger M (2003) Chemical ablation of sensory afferents in the walking system of the cat abolishes the capacity for functional recovery after peripheral nerve lesions. Exp Brain Res 150:50-60.
Quinn GP, Keough MJ (2002) Experimental design and data analysis for biologists, pp 537. Cambridge, UK: Cambridge UP.

Shelton PMJ, Stephen RO, Scott JJA, Tindall AR (1992) The apodeme complex of the femoral chordotonal organ in the metathoracic leg of the locust Schistocerca gregaria. J Exp Biol 163:345-358.

Stein W, Schmitz J (1999) Multimodal convergence of presynaptic afferent inhibition in insect proprioceptors. J Neurophysiol 82:512-514.

Usherwood PNR, Runion HI, Campbell JI (1968) Structure and physiology of a chordotonal organ in the locust leg. J Exp Biol 48:305-323.

Williamson R, Burns MD (1978) Multiterminal receptors in the locust mesothoracic leg. J Insect Physiol 24:661-666.

Wolf H, Büschges A (1997) Plasticity of synaptic connections in sensorymotor pathways of the adult locust flight system. J Neurophysiol $78: 1276-1284$

Zakotnik J, Matheson T, Dürr V (2004) A posture optimisation algorithm for model-based motion capture of movement sequences. J Neurosci Methods 135:43-54.

Zakotnik J, Matheson T, Dürr V (2006) Co-contraction and passive forces facilitate load compensation of aimed limb movements. J Neurosci 26: 4995-5007.

Zill SN (1985) Plasticity and proprioception in the insects. I. Responses and cellular properties of individual receptors of the locust metathoracic femoral chordotonal organ. J Exp Biol 11:435-461. 